\title{
Characterization of Fiber Direction Influence in CFRP Composites Using Advanced NDE Techniques
}

\author{
Kwang-Hee Im1,\#, Ju-Hwan Jang1, Chong-Gui Back1, Ok-Su Jeong1, and David K. Hsu² \\ 1 Department of Automotive Eng., WoosukUniversity 490, Hujung-ri, Samrae-up, Wanju-kun, Chonbuk, 565-701, Korea \\ 2 Center for Nondestructive Evaluation, lowa State University,IA 50011, USA \\ \# Corresponding Author / E-mail: khim@woosuk.ac.kr, TEL: +82+63-290-1473, FAX: +82+63-291-9312 \\ (Manuscript received: Sep, 16, 2012 / Revised: Nov, 15, 2012 / Accepted: Nov, 27, 2012)
}

\begin{abstract}
A nondestructive technique would be very useful. Advanced NDE T-ray (terahertz ray) techniques of technology and instrumentation has provided a probing field on the electromagnetic spectrum. However, the T-ray is limited in order to penetrate a conducting material to some degree. Here, the T-ray would not go through easily the CFRP composite laminates since carbon fibers are electrically conducting while the epoxy matrix is not. So, investigation of terahertz time domain spectroscopy (THz TDS) was made and reflection and transmission configurations were studied for a 48-ply thermoplastic PPS(poly-phenylene sulfide)-based CFRP solid laminate. It is found that the electrical conductivity of CFRP composites depends on the direction of unidirectional fibers.
\end{abstract}

KEYWORDS: CFRP composites, NDE, T-ray, C-scan image, Electrical conductivity

\section{Introduction}

Especially the importance of CF/Epoxy composites has been generally recognized $^{(1)}$, and CFRP composite laminates are widely used. Also, composites are currently being considered for many structural (aerospace vehicles, automobiles, trains and ships) applications due to their potential for reducing structural weight. Increasingly more high performance engineering structures are being built with critical structural components made from composite materials. So, $\mathrm{CF} /$ Epoxys are a material class for which nondestructive material property characterization is as important as flaw detection. Increasingly, a new NDE terahertz radiation has been recognized for their importance in technological applications. Recently, T-ray (terahertz ray) advances of technology and instrumentation has provided a probing field on the electromagnetic spectrum. The terahertz radiation is of critical importance in the spectroscopy evaluation of airport security screening, medical imaging, polar liquids, industrial systems and composites as well $^{(2)}$. Also the terahertz time domain spectroscopy (THz-TDS) is leading noncontact accurate detection of flaws and impact damages in composites, in which the THz-TDS is based on photoconductive switches, which rely on the production of few-cycle terahertz pulses using a femtosecond laser to excite a photoconductive antenna ${ }^{(3 \sim 4)}$. Another method is optical heterodyne conversion, or photomixing, which can be obtained using two continuous-wave (CW) lasers ${ }^{(5)}$. The mixing of two lasers could produce beating, which can modulate the conductance of a photoconductive switch by the terahertz difference frequency. So, the $\mathrm{CW}$-terahertz $(\mathrm{CW}-\mathrm{THz})$ radiation is produced.
So, the chemical property of materials is of great interest and the study presents many challenges. At the $\mathrm{THz}$ fundamental level, the problem is one of describing the overall properties of CFRP composite materials. In some cases, T-ray images can show chemical compositions of the object. These features of T-ray imaging have generated interest in commercial applications in diverse areas as moisture analysis, quality control of plastic parts and packaging inspection (monitoring) ${ }^{(6)}$. In this paper, we carried out our investigations of the index of

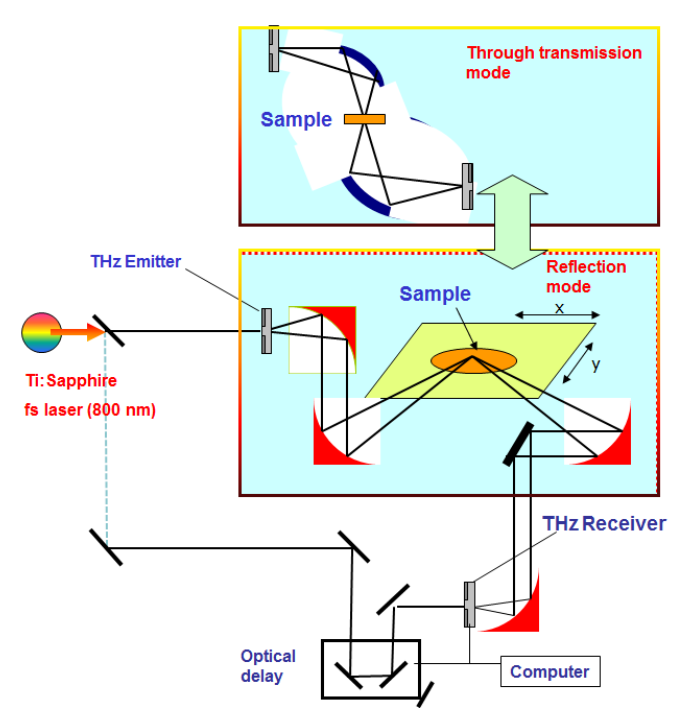

Fig 1. Schematic of the experimental setup used for terahertz transmission imaging 
refraction (n) and the absorption coefficient ( $\alpha$ ), the electrical conductivity of epoxy matrix composites containing carbon fibers and the THz scan images of CFRP solid laminates. Since carbon fibers are electrically conducting while the epoxy matrix is not, the CFRP composites are strongly anisotropic. Thus, our study is divided naturally into two parts. (1) The THz fundamental equations were derived in order to solve the index of refraction (n) in the general non-conducting materials. (2) The dependence of the effective conductivity on the fiber orientation is examined. The data confirm that these composites can be described by a conductivity model with principal axes along and normal to the nominal fiber axes. Thus, the $\mathrm{THz}$ effective scan images can be made according to the angels at the orientation of carbon fibers. It follows that the higher contrast images are being governed by the E-field direction.

\section{Experimental System}

\subsection{Configuration of System}

First of all, the terahertz instrumentation systems used in this research were provided by TeraView Limited (see Fig. 1). The instrumentation includes a time domain spectroscopy (TDS) pulsed system and a frequency domain continuous wave $(\mathrm{CW})$ system. The radiation emitted from a photoconducting antenna is broadband and spatially coherent, so it can be focused to a diffraction limited spot. As a result, it is possible to form images by translating an object through the focal spot of the $\mathrm{THz}$ beam, and recording the transmitted or reflected waveform as a function of the position of the object. The spatial resolution of a system of this type is determined by the spot size of the focused THz beam. This spot size is somewhat complicated, due to the broad bandwidth of the radiation, but a resolution below 0.5 $\mathrm{mm}$ is typical. In addition to the changes necessary for imaging, other advances have aided in the drive to develop THz-TDS as a viable commercial system by the TeraView Limited. The TDS system has a frequency range of $50 \mathrm{GHz}-4 \mathrm{THz}$ and a fast delay line up to $300 \mathrm{ps}$. Fig. 1 is a schematic diagram of a THz-TDS spectrometer, used for T-ray transmission imaging ${ }^{(6)}$. It consists of a femtosecond laser, a computer-controlled optical delay line, an optically gated terahertz transmitter, a set of off-axis paraboloidal mirrors for collimating and focusing the terahertz beam, the sample to be imaged, an opticallygated terahertz receiver, a current preamplifier, and a digital signal processor controlled by a personal computer.

\subsection{Measurement Techniques}

Refractive index could be considered one of properties in order to estimate materials as shown in Fig. 2; so we will suggest a procedure to estimate the electromagnetic properties such as the refractive index. Refractive index (n) for the through-transmission mode and

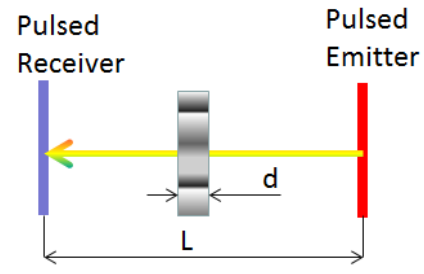

(a) Through-transmission mode

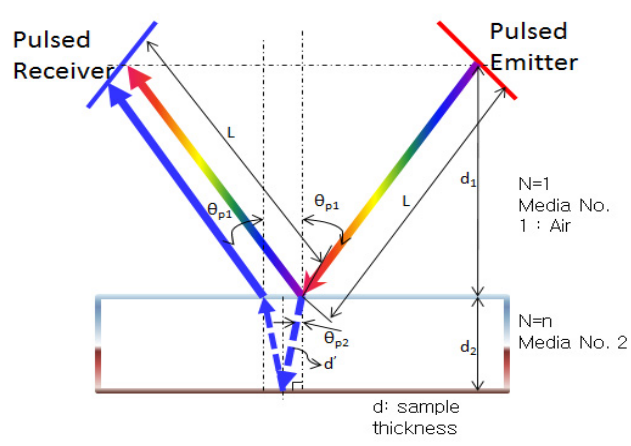

(b) Reflection mode

Fig. 2 Measurement of refractive index for T-ray applications

reflection mode can be solved as Eqs.(1) and (2) respectively ${ }^{(7)}$.

$$
\begin{aligned}
& \therefore n=1+\frac{\Delta t V_{a i r}}{d} \\
& n^{4}-A n^{2}-A \sin ^{2} \theta_{p 1}=0
\end{aligned}
$$

where $d$ is the sample thickness, $V_{\text {air }}$ is a light speed in air and $V_{s}$ is a light speed in sample, $\Delta \mathrm{t}(\mathrm{T})$ is the difference time between with sample and without sample and $A=\frac{T^{2} V_{\text {air }}^{2}}{4 d_{2}^{2}}$.

\section{Discussion and Results}

\subsection{Measurement of Refractive Index}

In preparation for measuring material parameters, we first measured a THz pulse reflected from PMMA, Fused quartz and GFRP composites. A method to determine the index of refraction was used to calculate the optical path length difference using the reflection and

Table 1 THz pulse reflected from PMMA, Fused quartz and GFRP composites

\begin{tabular}{|c|c|c|c|}
\hline \multirow{2}{*}{ Materials } & \multirow{2}{*}{$\begin{array}{c}\text { Refractive } \\
\text { index(n)* }\end{array}$} & \multicolumn{2}{|c|}{ Refractive index (n) } \\
\cline { 3 - 4 } & & Through-transmission mode & Reflection mode \\
\hline PMMA & $1.60 \pm 0.08$ & $1.61 \pm 0.07$ & $1.62 \pm 0.03$ \\
\hline Fused quartz & $1.95 \pm 0.05$ & $1.95 \pm 0.05$ & $1.94 \pm 0.05$ \\
\hline GFRP & - & $2.13 \pm 0.05$ & $2.12 \pm 0.05$ \\
\hline
\end{tabular}

*Referred to Reference [8] 


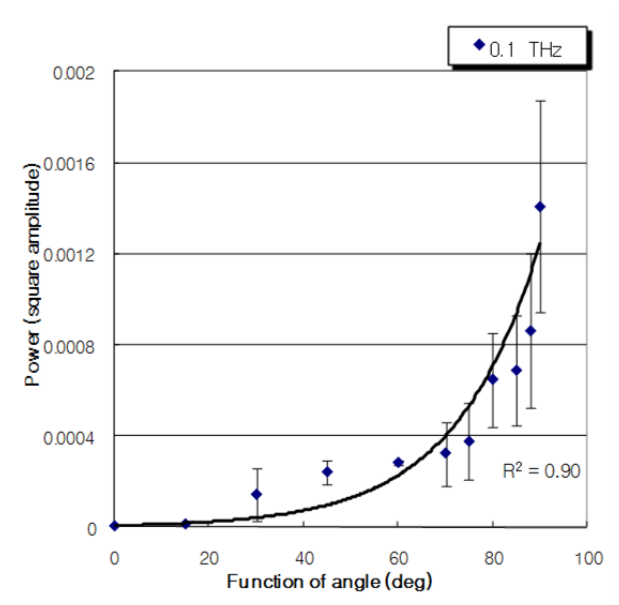

Fig. 3 Angular dependence of transmitted power of THz terahertz waves through a 12-ply unidirectional carbon composite laminate

through -transmission mode as shown in Table 1. It is found that those data were agreed with the known data for two modes.

\subsection{E-Field Characterization in Carbon Fiber}

Terahertz waves can penetrate dielectric materials quite easily but not electrically conducting materials. The application of terahertz waves to the inspection of carbon composites is mentioned in the literature ${ }^{(8)}$ but there has not been in depth studies. Carbon fiber reinforced polymer composites (CFRP) are poor conductors for electricity and the conductivity is anisotropic, so it is worthwhile to quantify the penetration of terahertz waves in carbon composites. The carbon fibers used in the manufacturing of CFRP are highly anisotropic microscopically; the electrical conductivity along the fiber axis is about three orders of magnitude greater than that in the radial direction. In a unidirectional laminate of carbon fiber composite, the transverse electrical conductivity is further impeded by the lack of continuity.

The conduction mechanism in the transverse direction (perpendicular to the fiber axis) is a percolation process that relies on the random contact between adjacent fibers. In the literature, the electrical conductivity data for carbon composites are somewhat sparse ${ }^{(9)}$. The reported values of longitudinal conductivity $\left(\sigma_{1}\right)$ varied from 1 x 104 $\mathrm{S} / \mathrm{m}$ to $6 \times 104 \mathrm{~S} / \mathrm{m}$. The range of reported data on the transverse conductivity $\left(\sigma_{t}\right)$ is particularly large; from $2 \mathrm{~S} / \mathrm{m}$ to as high as 600 $\mathrm{S} / \mathrm{m}$.

The value of transverse conductivity in a unidirectional laminate is highly dependent on the manufacturing process and the quality of the composite. In a unidirectional carbon composite, the in-plane conductivity with the electrical current flowing at an angle $\theta$ from the fiber axis is given by the references ${ }^{(9 \sim 10)}$ because of the highly anisotropic electrical conductivity $\left(\sigma_{1}>>\sigma_{t}\right)$,

Experimentally, we have measured the angular dependence of the power transmission through a 22-ply unidirectional carbon composite

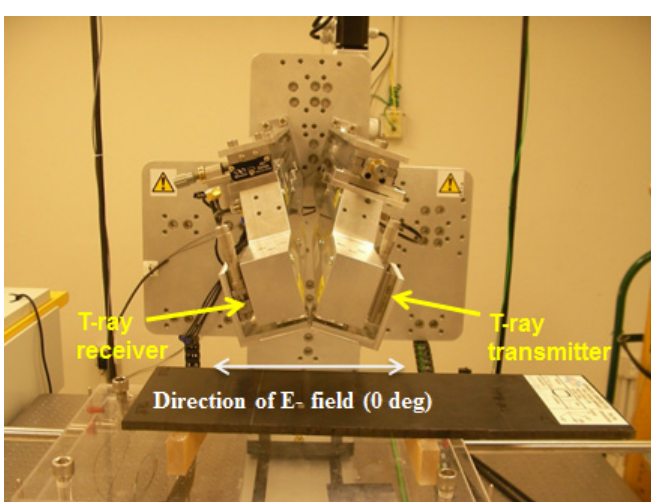

Fig. 4 Diagram showing a sample set up

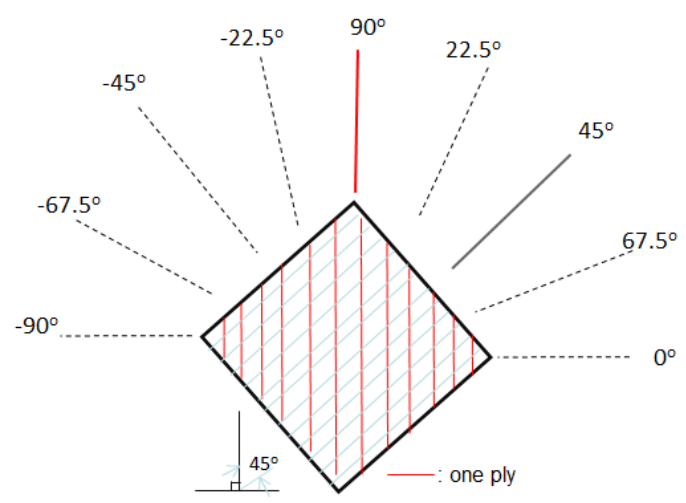

Fig. 5 Diagram showing a scan configuration of TDS system for scanning and direction of E-field normal to fiber and $\theta$ is the angle between fiber dir and E-field dir

laminate using the CW terahertz system. Near the low end of the frequency spectrum ( $\mathrm{f} \sim 0.1 \mathrm{THz}$ ), the transmitted power is more than $30 \mathrm{~dB}$ above the noise floor. The angular dependence of the transmitted power at $0.1 \mathrm{THz}$ is shown in Fig. 3. When compared to the theory prediction based on the angular dependent conductivity, the measured power transmission at angles away from 90 degree much higher the predicted as shown in Fig. 3.

\subsection{THZ Images in PPS CFRP Solid Laminate}

A reflective TDS system was utilized in order to evaluate the effect of E-filed direction on PPS CFRP solid laminate. A PPS CFRP solid laminate was scanned at different angles. Sample's layup is [45/0/-45/ $90]_{6 \mathrm{~s}}$ and $114 \times 355 \times 6.8 \mathrm{~mm}$ in size. Fig. 4 shows a sample set up of TDS system for scanning. Fig. 5 shows a scan configuration and direction of E-field normal to fiber and $\theta$ is the angle between fiber direction and E-field direction.

TDS scan images were made at the different time gates and best combination of E-field directions as shown in Fig. 6. These images were mapped out based on the E-field direction in the CFRP solid laminates. Scan size is $60 \times 60 \mathrm{~mm}$ and step size is $0.5 \mathrm{~mm}$. These 


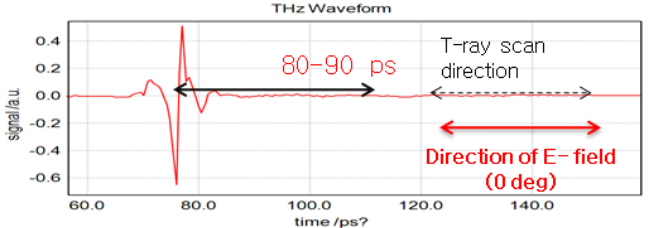

(a) A-scan
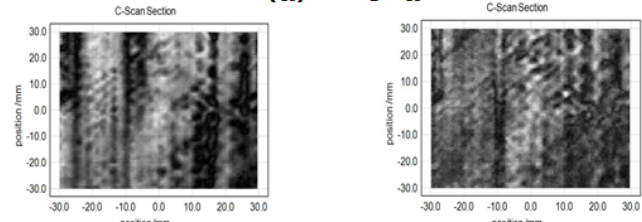

(b) $80-82 \mathrm{ps}$

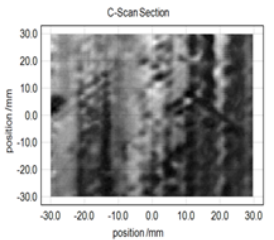

(d) $80-90 \mathrm{ps}$ (c) $83-85 \mathrm{ps}$

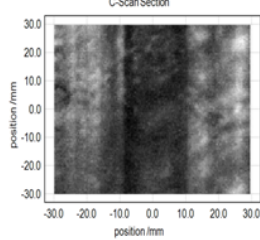

(e) $83-100 \mathrm{ps}$

Fig. 6 Diagram showing TDS scan images at the different time gates

image are based on a reflection mode at the scan range of $\Delta \mathrm{t}$ (scan gate length) $=10 \mathrm{ps}$. When a THz image was selected based on time gate and amplitude, it was found that all the same conditions be kept all the time during analyzing the images.

Fig. 7 shows a TDS typical scan images based on FFT at the angles $(\theta)$ of $-45^{\circ},-67.5^{\circ}, 45^{\circ}$ and $0^{\circ}$. Especially, at $\theta=-67.5^{\circ} \mathrm{o}$, this image to be considered clear and higher contrast as shown in Fig. 7(b). Here, there are some lines in the angle of $\theta=-67.5^{\circ}$ o due to the E-field

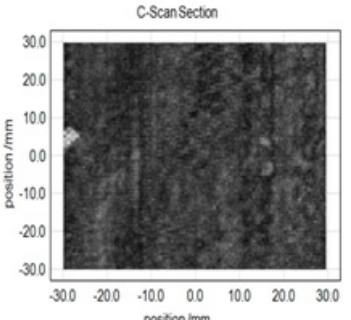

(a) $\Theta=-45.0^{\circ}$ c. Scansection

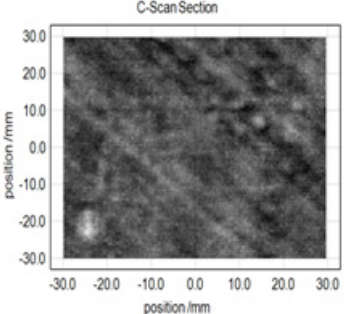

(c) $\theta=45.0^{\circ}$

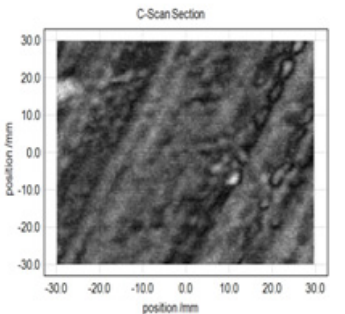

(b) $\Theta=-67.5^{\circ}$

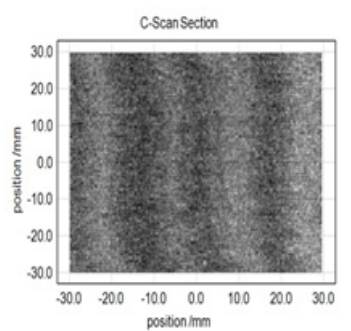

(d) $\theta=0^{\circ}$

Fig. 7 FFT diagrams showing a TDS typical scan images at the angles $(\theta)$ of (a) $-45^{\circ},\left(\right.$ b) $-67.5^{\circ}$, (c) $45^{\circ}$, and (d) $0^{\circ}$

direction of $\mathrm{THz}$ wave. It is thought that the $\mathrm{THz}$ wave penetrated a couple of a CFRP prepreg sheet called a scan depth.

\section{Conclusions}

The conductivity will substantially limit the utility of terahertz waves even in one- or two- ply PPS CFRP laminate. in conducting composites of CFRP composites especially with its penetration ability. Following conclusions are obtained as follows;

(1) It is found that the detectability of the flaw depends on the relation between the E-field and the fiber direction because of depending on the relative angle between the electrical field vector and the fiber axis.

(2) It was easier to inspect the material properties of the composite using a reflection configuration, which mode is certainly a more likely candidate for a maintenance technique.

(3) FFT scan images can be mapped out for the ply-layup orientation of the laminates at the surface based on the optimization of E-filed direction.

\section{ACKNOWLEDGMENTS}

This terahertz work was supported by Basic Science Research Program through the National Research Foundation of Korea(NRF) funded by the Ministry of Education, Science and Technology (2011-0008391) and also was helped by CNDE of Iowa State University.

\section{REFERENCES}

(1) Im, K. H., Park, N. S., and Yang, I. Y., 2001, "Characteristics of Falling Weight Impact Responses due to Stacking Sequences of CF/Epoxy Composite Plates," Journal of the Korean Society of Machine Tool Engineers, Vol. 10, No. 6, pp. 48 56.

(2) Huber, R., Brodschelm, A., Tauser, A., and Leitenstorfer, A., 2000, "Generation and Field-resolved Detection of Femtosecond Electromagnetic Pulses Tunable up to 41 THz," Appl. Phys. Lett., Vol. 76, No. 1, pp. 3191 3199.

(3) Rudd, J. V., and Mittleman, D. M., 2000, "Influence of Substrate-lens Design in Terahertz Time-domain Spectroscopy," J. Opt. Soc. Amer. B, Vol. 19, No. 2, pp. 319 329.

(4) Gregory, I. S., Baker, C., Tribe, W., Bradley, I. V., Evans, M. J., and Linfield, E. H., 2000, "Optimization of Photomixers and Antennas for Continuous-wave Terahertz Emission," IEEE Journal Of Quantum Electronics, Vol. 41, No. 5, pp. 71 728.

(5) Brown, E. R., Smith, F. W., and McIntosh, K. A., 1993, "Coherent Millimeterwave Generation by Heterodyne Conversion in Low-temperature-grown GaAs Photoconductors," J. Appl. Phys., Vol. 73, No. 3, pp. 1480 1484. 
(6) Brown, E. R., McIntosh, K. A., Nichols, K. B., and Dennis, C. L., 1995, "Photomixing up to $3.8 \mathrm{THz}$ in Low-temperaturegrown GaAs," Appl. Phys. Lett., Vol. 66, No. 3, pp. 285 287.

(7) Im, K. H., Kim, J. H., and Hsu, D. K., 2012, "Nondestructive Evaluation in the Defects of FRP Composites by using Terahertz Waves," Journal of the Korean Society of Manufacturing Technology Engineers, Vol. 21, No. 2, pp. 252 258.

(8) Schueler, R., Joshi, S. P., and Schulte K., 2001, "Damage
Detection in CFRP by Electrical Conductivity Mapping," Composite Science and Technology, Vol. 61, No. 6, pp. 921 930.

(9) Hsu, D. K., 1985, "Characterization of a Graphite/Epoxy Laminate by Electrical Resistivity Measurements," Rev. Prog. in Quantitative NDE, Vol. 4, No.1, pp. 1219 1228.

(10) Tse, K. W., Moyer, C. A., and Arajs, S., 1981, "Electrical Conductivity of Graphite Fiber epoxy Resin Composites," Materials Science and Engineering, Vol. 49, No.1, pp. 41 46. 